\title{
Functional and structural abnormalities in the nerves of Type I diabetic baboons: aminoguanidine treatment does not improve nerve function
}

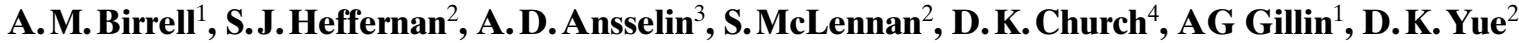 \\ ${ }^{1}$ The Departments of Renal Medicine, Royal Prince Alfred Hospital, Sydney, Australia \\ ${ }^{2}$ The Departments of Endocrinology, The University of Sydney, Australia \\ ${ }^{3}$ The Electron Microscopy Unit, The University of Sydney, Australia \\ ${ }^{4}$ The Department of Veterinary Clinical Science, The University of Sydney, Australia
}

\section{Abstract}

Aims/hypothesis. To improve understanding of the pathophysiology of diabetic neuropathy and to establish a primate model for experimental studies, we examined nerve changes in baboons with Type I (insulin-dependent) diabetes mellitus. We also examined the effect of aminoguanidine (an inhibitor of the formation of advanced glycation end products) on nerve function.

Methods. Male baboons (Papio hamadryas) were assigned to four groups; control, diabetic, control and diabetic treated with aminoguanidine. Diabetes was induced with streptozotocin (60 mg/kg, intravenous). Insulin and aminoguanidine $(10 \mathrm{mg} / \mathrm{kg})$ were injected subcutaneously daily. Motor and sensory nerve conduction velocity was measured using standard techniques. Autonomic function was examined by measuring heart rate response to positional change. Sural nerve morphometry was analysed in the diabetic group (mean duration 5.5 years) along with their age-matched controls.

Results. The diabetic groups were smaller in size with a mean $\mathrm{HbA}_{1 \mathrm{c}}$ of $8.9 \pm 1.2 \%$. The nerve conduction velocity and heart rate response was reduced in the diabetic groups. Morphometric analysis of the diabetic sural nerve showed smaller axon diameter $(2.99 \pm 0.06 \mu \mathrm{m}$ vs $3.29 \pm 0.06 \mu \mathrm{m} ; p<0.01)$ accompanied by thinner myelin $(1.02 \pm 0.02 \mu \mathrm{m}$ vs $1.15 \pm 0.02 \mu \mathrm{m}, p<0.01)$ with no change in the axon density. Treatment with aminoguanidine for 3 years had no effect on glycaemic control and did not restore conduction velocity or autonomic dysfunction in the diabetic animals, contrary to the studies in rats. Conclusions/interpretation. These results show that the primate is a good model to study diabetic neuropathy and suggest that the accumulation of advanced glycation end products are not an early mechanism of nerve damage in this disorder. [Diabetologia (2000) 43: 110-116]

Keywords Diabetic complications, neuropathy, autonomic, nerve conduction velocity, aminoguanidine, monkey, morphometry, myelin, advanced glycation end products.
Diabetic neuropathy is a serious complication affecting as many as $60 \%$ of diabetic patients [1]. Its clini-

Received: 24 June 1999 and in revised form: 23 August 1999

Corresponding author: A. Birrell, Department of Renal Medicine, Royal Prince Alfred Hospital, Missenden Rd, Camperdown, NSW 2050 Australia

Abbreviations: MNCV, Motor nerve conduction velocity; SNCV, sensory nerve conduction velocity; AGE, advanced glycation end products; STZ, streptozotocin. cal manifestations can vary from reduced nerve conduction velocity and sensory deficits to autonomic disturbances associated with increased mortality [2, 3]. Despite numerous research efforts, the pathogenesis of diabetic neuropathy remains incompletely understood. Proposed mechanisms include microvascular abnormalities, altered Schwann cell or neuronal metabolism, deficiencies of neurotrophic substances and slowing of axonal transport [4-7]. In diabetic patients, changes in function of peripheral and autonomic nerves can be detected very early in the dis- 
ease. There is a characteristic slowing of both motor and sensory nerve conduction velocity which improves with better metabolic control $[6,8]$. Abnormalities of heart rate variation have also been reported $[2,9]$. These functional abnormalities precede overt symptoms by many years. In the more advanced phase of diabetes, sensory symptoms and autonomic disturbance often become clinical problems. At this stage, nerve conduction and heart rate variation are usually abnormal and accompanied by pathological changes of demyelination, decrease in axon diameter, fibre loss and nodal swelling [10-12].

Much less is known about the very early structural changes in patients with diabetes as biopsy specimens are rarely available [13]. In the rat, several studies have reported electrophysiological and structural changes occurring within months of induction of diabetes but the differences in anatomy and physiology of rats from humans make this model not entirely satisfactory for studying the human disease $[5,14]$. Therefore we examined nerve changes in the first 5 years of diabetes in our established colony of baboons with Type I (insulin-dependent) diabetes mellitus.

In addition, a group of diabetic and control animals treated with aminoguanidine were studied. It has been proposed that the excess formation of advanced glycation end products (AGEs) in diabetes causes cellular dysfunction and damage, leading to diabetic complications [15, 16]. Aminoguanidine has been shown to inhibit the formation of AGEs in tissues including the nerve $[17,18]$. A number of studies have reported that treatment with aminoguanidine in diabetic rats partially restored nerve conduction velocity and was associated with preservation of axonal size [18-22]. Our aim was to examine the effects of aminoguanidine on the functional nerve changes in the baboon in the early stages of diabetes.

\section{Materials and methods}

Animals. Male baboons (Papio hamadryas) were used in a long-term diabetic study and the husbandry of these animals has been reported previously [23]. In brief, the animals were housed in an outdoor enclosure at the National Baboon Colony in accordance with the guidelines of the Institutional Animal Welfare Committee, Animal Research Review Panel and the National Health and Medical Research Council [24, 25]. The baboons were fed twice daily at times that corresponded to peak insulin concentrations. The diet consisted of a ration of bread, fruit and vegetables, primate pellets (Doust and Rabbidge, NSW, Australia), nuts and seeds. They had free access to water. Baboons were assigned randomly at 2.5 years of age $(5 \mathrm{~kg})$ to controls $(n=8)$, diabetic $(n=10)$, diabetics treated with aminoguanidine $(n=5)$ and controls treated with aminoguanidine $(n=5)$. Due to a limitation of the numbers of animals of the same age, it was necessary for an aminoguanidinetreated control group to be started at 3 years of age $(7.5 \mathrm{~kg})$. Diabetes was induced by an intravenous injection of freshly prepared streptozotocin (STZ, Sigma, NSW, Australia) $(60 \mathrm{mg} / \mathrm{kg}$, in $0.1 \mathrm{~mol} / \mathrm{l}$ citrate buffer, $\mathrm{pH} 4.5)$. After several days of consistently high fasting plasma glucose concentrations ( $>25 \mathrm{mmol} / \mathrm{l}$ ), the animals were started on a once (given between 0800-0900 hours) daily injection of long and short acting insulin with dosage titrated to maintain moderate hyperglycaemia. Each month the animals were weighed and had blood taken for biochemistry and haematology. Plasma glucose was measured by glucose oxidase and glycated haemoglobin was measured by high performance liquid chromatography (Bio-Rad, Richmond, Calif., USA). All procedures were carried out under ketamine anaesthesia (Ketalar, Parke Davis, NSW, Australia) either intramuscularly $(6 \mathrm{mg} / \mathrm{kg})$ for short procedures or intravenously $\left(13 \mathrm{mg} \cdot \mathrm{kg}^{-1} \cdot \mathrm{h}^{-1}\right)$ for longer ones.

The aminoguanidine treated groups received daily (between 0800 and 0900 hours) subcutaneous injections of aminoguanidine at $10 \mathrm{mg} / \mathrm{kg}$ (supplied by Alteon, N.J., USA) from the time they entered the study. The concentration of aminoguanidine in the blood of control and diabetic animals was measured as described previously [26]. Otherwise the aminoguanidine-treated animals were handled identically to the untreated groups.

Nerve conduction velocity. Motor nerve conduction velocity (MNCV) and sensory nerve conduction velocity (SNCV) were measured in the sciatic and sural nerves (respectively) of each experimental animal, using methods modelled on those described for humans [27]. The skin temperature of the animals was monitored by a thermistor (Digi-Sense, Model 8528-40, Cole-Parmer Instrument Co, NSW, Australia), placed on the sole of the foot and maintained at or above $36^{\circ} \mathrm{C}$. The nerves were stimulated with 27 gauge needle electrodes, using square wave impulses of $0.5 \mathrm{~ms}$ duration generated at 2 pulses per second by the MS91 (Medelec, Surrey, UK). For MNCV, compound muscle action potentials were measured from the dorsal surface of the foot after stimulation from the sciatic notch and the popliteal fossa. The interelectrode distance was measured to the nearest millimeter and used to eliminate variability from the neuromuscular junction. The SNCV was measured orthodromically in each sural nerve. Thirty two traces were recorded and averaged by the MacLab (Analog Digital Instruments Pty., Coopertino, Calif., USA) to eliminate noise. All results were stored and analysed on the MacLab. The MNCV and SNCV were calculated for both legs and the mean used as the conduction velocity.

To define more precisely the effects of age on nerve conduction, 19 male animals (aged 1 year to 8 years) from the colony had MNCV and SNCV measurement. As there is no published data on conduction velocity in different ages of baboons, it was necessary to establish our own values over that period.

Autonomic function. Autonomic function was assessed by measuring the heart rate response to postural change when the mean duration of diabetes was $3.9 \pm 1.7$ years. A regimen of bolus intramuscular ketamine injection $(6 \mathrm{mg} / \mathrm{kg})$ followed by low dose intravenous infusion $\left(0.13 \mathrm{mg} \cdot \mathrm{kg}^{-1} \cdot \mathrm{h}^{-1}\right)$, was used to stabilise the heart rate before beginning the study as described previously [28]. Ketamine was selected as the anaesthetic agent to minimise the effects on the cardiovascular system $[28,29]$. After the initial sedation, there was a 15-min period to stabilise the heart rate. This was followed by $5 \mathrm{~min}$ of recording before and after the animal was positioned upright on a tilt table specially designed for similar work in humans. The heart rate variation was calculated as the percentage change in beats/min in the erect and supine position. 
Table 1. Clinical variables of the baboons

\begin{tabular}{|c|c|c|c|}
\hline & $\begin{array}{l}\begin{array}{l}\text { Duration } \\
\text { of diabetes } \\
\text { (years) }\end{array} \\
\text { (y) }\end{array}$ & $\begin{array}{l}\text { Fasting } \\
\text { plasma glucose } \\
(\mathrm{mmol} / \mathrm{L})\end{array}$ & $\mathrm{HbA}_{1 \mathrm{c}}(\%)$ \\
\hline Control $(n=8)$ & - & $4.8 \pm 0.4$ & $4.6 \pm 0.4$ \\
\hline Diabetic $(n=10)$ & $4.8 \pm 1.7$ & $21.6 \pm 5.1^{\mathrm{a}}$ & $8.9 \pm 1.2^{\mathrm{a}}$ \\
\hline $\begin{array}{l}\text { Control + amino- } \\
\text { guanidine }(n=5)\end{array}$ & - & $4.9 \pm 0.3$ & $4.6 \pm 0.1$ \\
\hline $\begin{array}{l}\text { Diabetic + amino- } \\
\text { guanidine }(n=5)\end{array}$ & $2.9 \pm 0.1$ & $23.4 \pm 2.4^{\mathrm{a}}$ & $8.5 \pm 0.9^{\mathrm{a}}$ \\
\hline
\end{tabular}

Values are in means $\pm \mathrm{SD}$. ${ }^{\text {a }} P<0.001$ compared with respective control animals

Nerve morphometric analysis. A biopsy specimen was taken from the right sural nerve and processed for transmission electromicroscopy (TEM). The nerve section was fixed in $2 \%$ acrolein and $2 \%$ gluteraldehyde in $0.1 \mathrm{~mol} / 1$ phosphate buffer at $\mathrm{pH} 7.3$ and post fixed in $1 \%$ osmium tetroxide in $0.1 \mathrm{~mol} / \mathrm{l}$ phosphate buffer. Specimens were dehydrated, infiltrated and embedded in Spurr's resin. Thin sections were cut on an Ultracut (Leica, Vienna, Austria) and mounted onto 400 mesh copper grids, stained with uranyl acetate solution and lead citrate. Sections were viewed on a CM12 (Philips, Eindhoven, The Netherlands) and digital electron micrographs $(\times 4400)$ were recorded on a Macintosh computer with a Digital Micrograph program (Gatan, Pleasanton, Calif., USA). We studied seven diabetic animals with a mean diabetic duration of $5.2 \pm$ 0.8 years and seven age-matched controls. Each biopsy specimen usually contained three to five fascicles and all the fibres not obscured by grid bars were measured. Myelinated axons were measured for axon diameter and myelin thickness using the PrismView program (Analytical Vision, Raleigh, N.C., USA) on a Macintosh computer. Axon counts were estimated by counting all profiles not obscured by the grid and calculated as number of axons per $\mathrm{mm}^{2}$ and corrected for edge effect [31]. The $g$ ratio of the fibres, which is myelin sheath thickness divided by the axon diameter, was calculated for each myelinated axon. The observer was blinded to the diabetic status of the specimens.

Statistical analysis. All data are presented as mean \pm standard deviation (SD). Statistical significance between groups was analysed using ANOVA. A $p$ value of less than 0.01 was considered significant.

\section{Results}

Biochemical and clinical profile. The STZ-induced diabetic animals showed consistent fasting hyperglycaemia throughout the study (Table 1 ). Insulin treatment maintained a mean $\mathrm{HbA}_{1 \mathrm{c}}$ in the diabetic groups similar to that seen in humans with Type I diabetes. The baseline weight at the beginning of the study was similar in all groups. The diabetic groups had, however, a slower growth rate and at 6 years were considerably less in weight (Fig. 1).

Nerve conduction studies. The electrophysiological results are shown in Figures 2 and 3. In the controls,

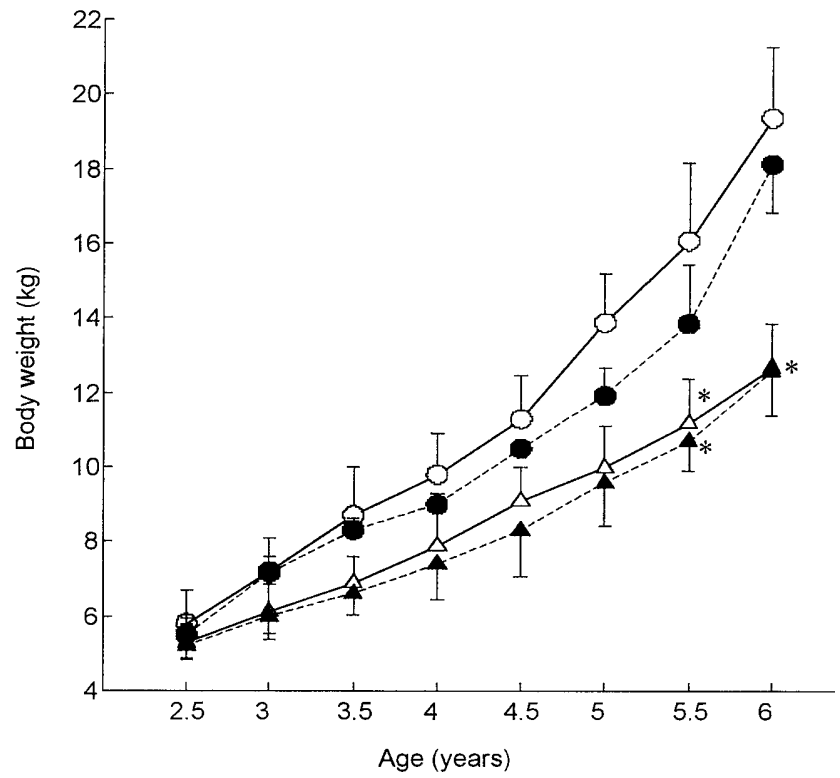

Fig. 1. The weights of the control animals $(\bigcirc)$, controls treated with aminoguanidine $(O)$, diabetics $(\Delta)$ and diabetics treated with aminoguanidine $(\boldsymbol{\Delta})$ from 2.5 years of age (start of the study) to 6 years (4.5 years duration of diabetes). Error bars are SD. ${ }^{*} p<0.001$ from the controls

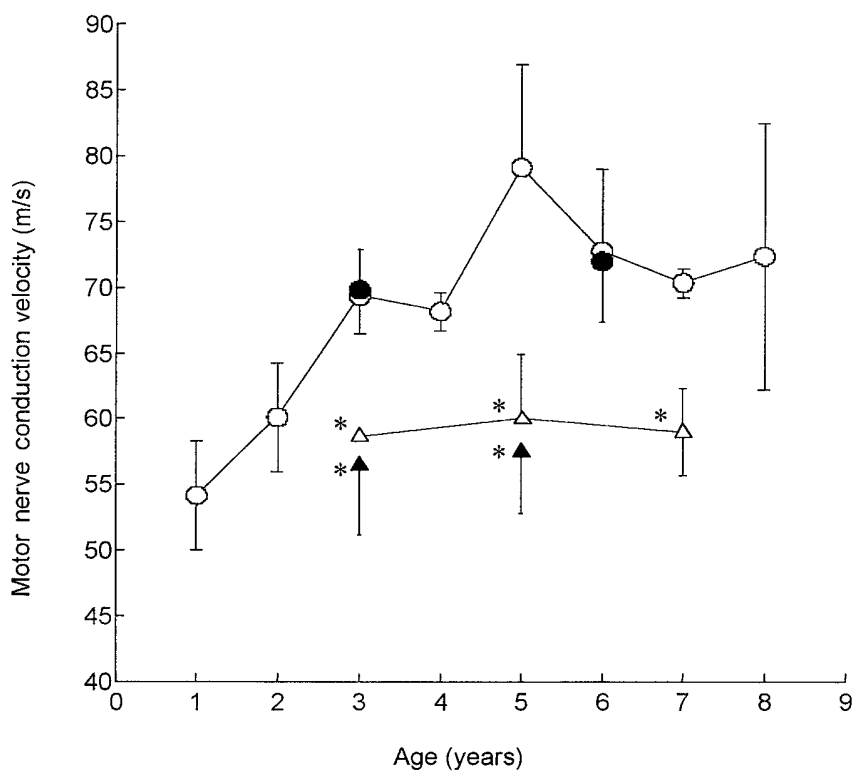

Fig. 2. The change of MNCV with age (years) in the controls $(\bigcirc)$, controls treated with aminoguanidine $(O)$, diabetics $(\Delta)$ and diabetics treated with aminoguanidine $(\boldsymbol{\Delta})$. Error bars are SD. $* p<0.01$ from the controls

MNCV and SNCV showed a steep increase from 1 to 3 years of age, thereafter the curve plateaus. The diabetic group had a significantly lower MNCV and SNCV $(p<0.01)$. The amplitude of the sensory evoked potential was not significantly different between the control and diabetic animals $(0.25 \pm 0.08$ and $0.27 \pm 0.07 \mu \mathrm{V}$, respectively). 


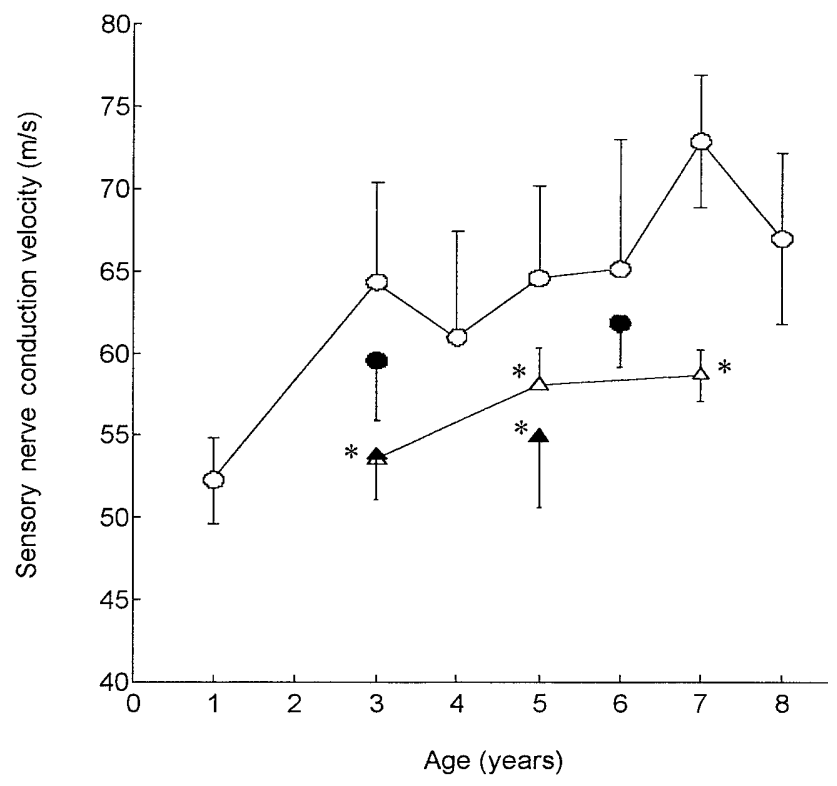

Fig. 3. The change of SNCV with age (years) in the controls $(\bigcirc)$, controls treated with aminoguanidine $(\mathcal{O})$, diabetics $(\Delta)$ and diabetics treated with aminoguanidine $(\boldsymbol{\Delta})$. Error bars are SD. $* p<0.01$ from the controls

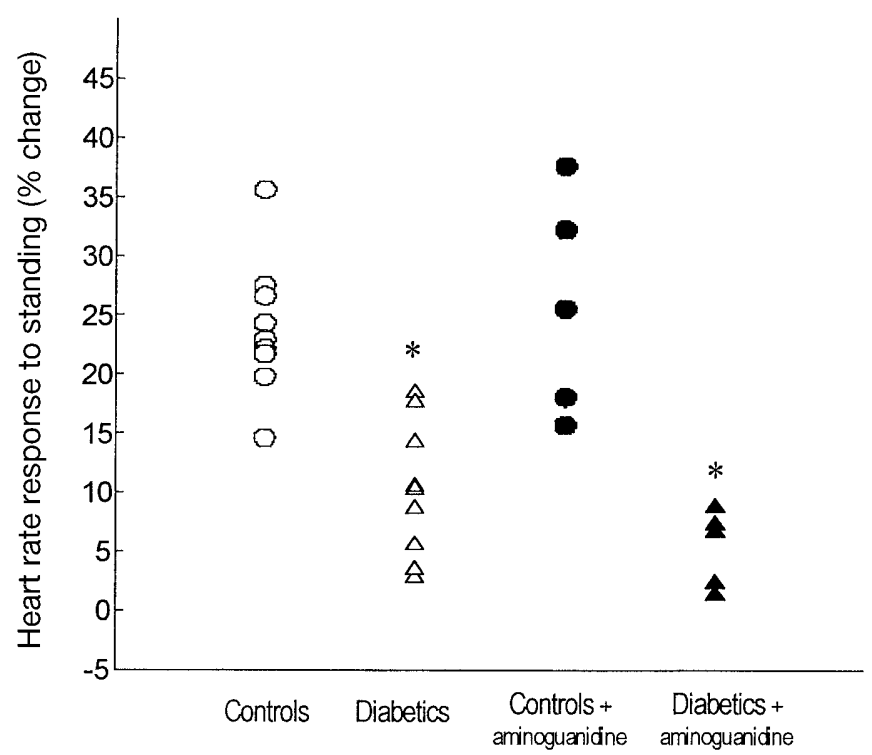

Fig.4. Heart rate regulation in response to change in position from supine to upright in the four groups. The response is in percentage change of heart rate from the supine position. $* p<0.01$ from the controls

Autonomic function. The autonomic function test showed that the diabetic group had an attenuated increase in heart rate response when moved upright from the supine position (Fig. 4).

Morphometric analyses. The diabetic group had a mean myelinated axon diameter $(2.99 \pm 0.06 \mu \mathrm{m})$ which was significantly lower than the control group

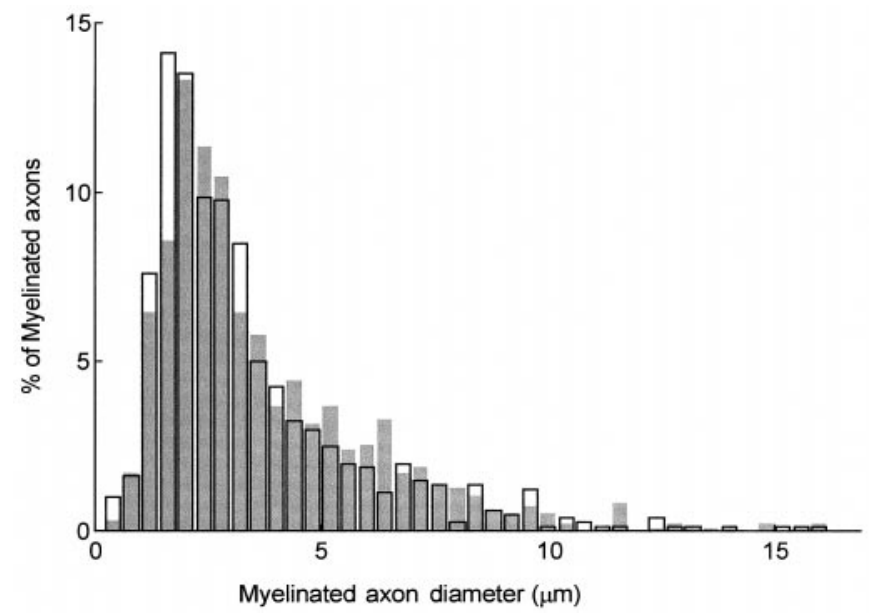

Fig. 5. Frequency histogram of the myelinated axon diameter in control and diabetic groups. $\square$ represents the percentage of myelinated axons at each axon diameter for the control animals. $\square$ represents the values of the diabetic animals

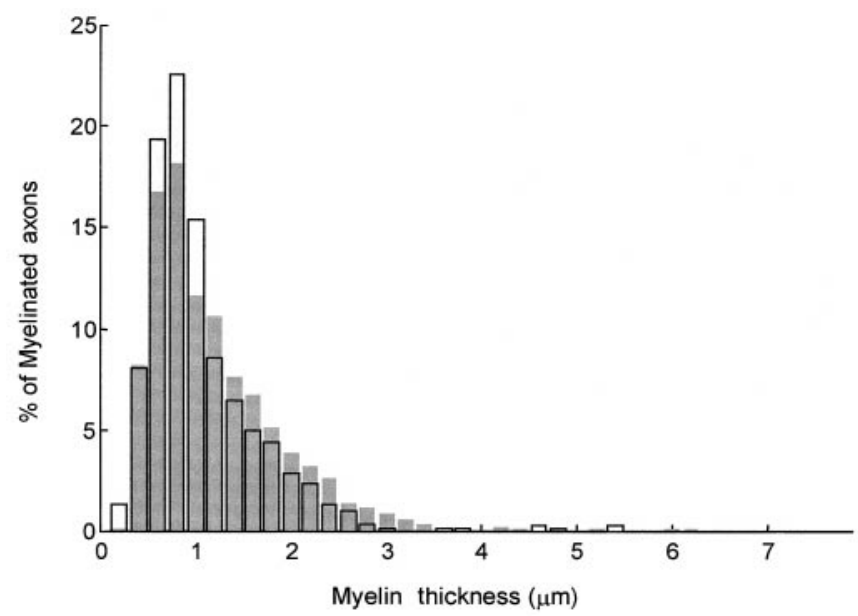

Fig. 6. Frequency histogram of the axon myelin thickness in control and diabetic groups. $\square$ represents the percentage of myelinated axons at each myelin width for the control animals. represents the values of the diabetic animals

$(3.29 \pm 0.06 \mu \mathrm{m} ; p<0.01)$. In the diabetic animals $24 \%$ of axons were less than $1.8 \mu \mathrm{m}$, whereas only $17 \%$ axons of control groups fell below this diameter. This is accompanied by a reduction of larger fibres (13\% between $4.4 \mu \mathrm{m}$ and $6.8 \mu \mathrm{m}$ for the diabetics compared with $20 \%$ for the controls) (Fig. 5). The distribution of myelin thickness of the diabetic nerve fibres showed a similar shift, the mean myelin thickness was significantly lower in the diabetic animals than controls $(1.02 \pm 0.02 \mu \mathrm{m}$ vs $1.15 \pm 0.02 \mu \mathrm{m}$, $p<0.01$ ) (Fig. 6). There was no significant difference between the $\mathrm{g}$ ratios of the diabetic $(0.45 \pm 0.1)$ and control $(0.39 \pm 0.1)$ groups nor any significant difference in nerve fibre density for the diabetic $(0.48 \pm$ $\left.0.22 \times 10^{3} \mathrm{~mm}^{-2}\right)$ and the controls $\left(0.51 \pm 0.21 \times 10^{3}\right.$ $\mathrm{mm}^{-2}$ ) groups. 


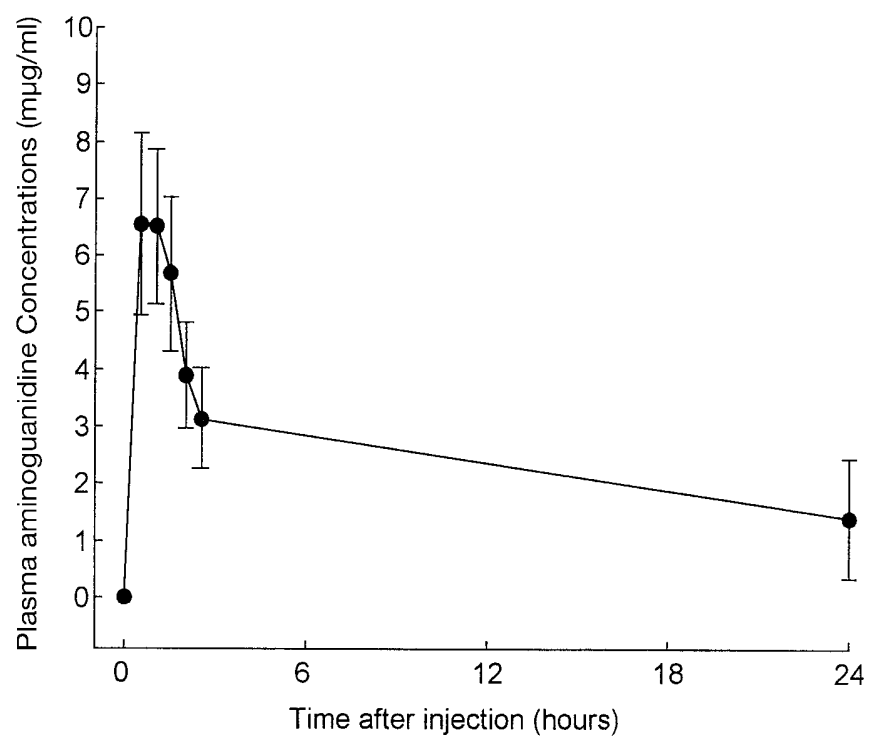

Fig. 7. Aminoguanidine concentration in the plasma of control and diabetic baboons after a subcutaneous injection of aminoguanidine $(10 \mathrm{mg} / \mathrm{kg}, n=9)$

Aminoguanidine treatment. Pharmacokinetic study showed that aminoguanidine injected according to our protocol increased plasma aminoguanidine concentration within $30 \mathrm{~min}$ which remained detectable at $24 \mathrm{~h}(1.4 \pm 1.06 \mu \mathrm{g} / \mathrm{ml})$ (Fig. 7). Treatment had no effect on glycaemic control (Table 1). Although the aminoguanidine-treated controls and diabetic groups were slightly lighter than their respective untreated groups, the difference never reached statistical significance (Fig. 1). The MNCV and SNCV of the diabetic animals were not restored by 3 years of aminoguanidine treatment and there was no statistically significant effects on the control MNCV and SNCV treated with aminoguanidine (Fig.2, 3). Aminoguanidine treatment had no effect on amplitude of the sensory action potential in the diabetic and control groups that were treated $(0.25 \pm 0.06$ and $0.26 \pm 0.1 \mu \mathrm{V}$, respectively) and did not alter the autonomic heart rate response to postural change (Fig. 7).

\section{Discussion}

Due to the difficulties of obtaining tissue samples in humans, there is still an incomplete knowledge of the pathogenesis and early changes in diabetic neuropathy. Primates have been used previously to examine complications of diabetes; their phylogenetic closeness to humans makes it easier to draw analogies between the species [23, 32-34]. The longevity of the primate permits long-term serial studies which are not possible in the rodent, the traditional experimental model of diabetes. This led us to document in a baboon model of Type I diabetes the functional and structural changes of the peripheral nerve in the first few years after induction of diabetes. Our results showed that there is a definite reduction in MNCV and SNCV by 3 years of diabetes with demonstrable morphometric changes at 5 years. Similar changes of nerve conduction have been reported in rats but as diabetes is usually induced in a phase of rapid body growth, it is difficult to distinguish the effects of diabetes from that of retarded maturation in this model $[5,14]$. This problem has been minimized in our study; the longevity of baboons allowed the induction of diabetes to be at an age when the increase in nerve conduction had plateaued, as shown by the measurements on the controls (Fig. 2, 3).

Another advantage of the primate model is the availability of samples for examination of nerve fibre structure in the first few years of diabetes. In the sciatic nerves of diabetic rats, there are obvious morphological changes within 5 months of induction [18, 35]. Interpretation of these results needs, however, to take into account that these rats are often extremely hyperglycaemic and in the rapid stage of nerve growth $[14,36]$. In humans, sural nerve biopsy specimens taken in the later stages of diabetes, usually when neuropathy is a clinical problem, have provided information on structural abnormalities. The loss of large axons, a shift towards smaller myelinated fibres and demyelination are characteristic findings $[10,12$, 37]. By comparison, there is little information on the changes in the first few years. The results of sural nerve biopsies from eight children with subtle neurophysiological changes (a reduction in NCV and amplitude), when the mean duration of diabetes was 8.5 years have been presented in abstract form [13]. Data from teased nerve fibres showed segmental demyelination and paranodal abnormalities but no axonal degeneration in diabetic subjects. These changes were not accompanied by alterations in myelinated fibre density, myelinated fibre area and axonal area. The duration of diabetes of our diabetic baboons was 5.2 years and the metabolic control was typical of young Type I diabetic patients. Morphometric studies in the sural nerves showed reduced myelin thickness parallelled by a commensurate fall in mean axon diameter. Although there are differences in the findings reported in the abstract and ours, both support the notion that in patients receiving conventional therapy, nerve changes are already evident within a few years of Type I diabetes. Only one other group have studied nerve morphology in a primate model of Type I diabetes [33]. They examined the sural nerve of macaques 12 to 36 months after induction of diabetes with STZ. They found mild changes to both myelinated and non-myelinated axons and concluded that due to the slow onset and mildness of pathology, this primate model was inferior to that of the rat for study of diabetic neuropathy. The findings reported in the abstract and ours indicate, however, that development of neuropathy in humans is indeed 
slow. In comparison to the relatively minor changes to morphology, nerve conduction velocities are much more affected. This dichotomy is typical of diabetic neuropathy and clearly indicates other factors affect nerve conduction, probably subtle biochemical or vascular changes or both. Morphometric changes of endoneurial blood vessels are also thought to be important in the pathogenesis of diabetic neuropathy and are currently being studied in these samples. The baboon model in our opinion appears to be appropriate for monitoring the development of neuropathy and the testing of treatment strategies in humans.

Clinically, autonomic neuropathy is a late complication in diabetes associated with high morbidity and mortality. Subtle changes of autonomic function can, however, be shown many years earlier [2]. There are two reports of a reduced heart rate response to postural change and deep breathing in $30 \%$ of children with diabetes of less than 10 years duration [9, 38]. Although structural changes of the autonomic nerve have been shown in diabetic rats, there has not been any suitable animal model for studying autonomic dysfunction [21]. The diabetic baboons showed a decrease in heart rate response similar to that seen in humans. Therefore, the baboon model of diabetes is also appropriate for studying the pathogenesis and treatment of autonomic neuropathy.

Advanced glycation end products have been found in the nerves of diabetic rats and have been postulated to have a pathogenetic role in neuropathy. They can disturb cell function in a number of ways, amongst these are effects on cross-linking of molecules, nitric oxide synthesis and action, generation of cytokines and production of free radicals [16]. Aminoguanidine inhibits the formation of AGEs and possibly prevent diabetic complications through one or more of these pathways. The trials of aminoguanidine treatment in humans with late stage diabetic complications are ongoing, although preliminary evidence indicates that it is not effective in advanced renal disease [39]. In our study of long-term aminoguanidine treatment in diabetic primates aiming at the prevention of diabetic complications in the early phase, both groups of baboons treated with aminoguanidine had similar glycaemic control but slightly lower weight gain than their untreated counterparts, a phenomenon also observed in rats [21, 40]. Despite 3 years of treatment from the onset of diabetes, aminoguanidine did not prevent the impairment of MNCV, SNCV or heart rate response of diabetic animals. Its effects on nerve morphometry have not been assessed at this stage because aminoguanidine treated animals have not reached the duration of diabetes for nerve biopsies electrophysiological results lead us, however, to believe that aminoguanidine will have little it any effect on structural changes. The inability of aminoguanidine to restore nerve function in baboons differs from some of the studies in rats that showed treatment to be beneficial although the results are conflicting [18, 19, 22, 41, 42]. One study found that diabetic rats given $50 \mathrm{mg} / \mathrm{kg}$ aminoguanidine in the drinking water for 16 weeks had NCV restored although doses of 10 and $25 \mathrm{mg} /$ $\mathrm{kg}$ were ineffective [19]. Another that diabetic rats injected intraperitoneally with aminoguanidine $\left(50 \mathrm{mg} \cdot \mathrm{kg}^{-1} \cdot\right.$ day $\left.^{-1}\right)$ had $\mathrm{NCV}$ restored but not when aminoguanidine $(1 \mathrm{~g} / \mathrm{l})$ was given in drinking water [22]. Yet another reported, however, that aminoguanidine $(1 \mathrm{~g} / \mathrm{l})$ in the drinking water did restore NCV in diabetic animals [40]. Apart from inhibiting AGEs formation, aminoguanidine has other actions which could be different in the rodents and primates. Due to the anatomy of rats, measurement of NCV is more difficult than in larger animals. These are all factors that may contribute to the differing results. The dosage of aminoguanidine used for our study was shown by pharmacokinetic studies to attain a plasma concentration clearly detectable after $24 \mathrm{~h}$ and similar to that reached in humans and rats at an equivalent dose [43, 44]. To be completely certain that aminoguanidine treatment in our hands has been effective in preventing formation of AGEs, it is necessary to measure these products in peripheral nerves. This is difficult due to the limited size of tissue samples but we are currently measuring the level of AGEs in renal biopsy samples of these baboons.

There has been much research on functional nerve changes in the early stages of diabetic neuropathy. There is, however, little information on early structural changes that could be the key to understand the long-term damage and the development of clinical disease. In this study we were able to examine the function of the peripheral and autonomic nerve in light of the morphometric changes of the sural nerve in the first 5 years of diabetes. The inability of aminoguanidine to prevent the decrease in nerve conduction velocity in the primate suggests that formation of AGEs is not the major cause of functional nerve changes in the early stages of diabetes.

Acknowledgements. This study was supported by the Rebecca Cooper Research Foundation, with assistance from the National Health and Medical Research Council (NHMRC) and the Juvenile Diabetes Foundation, International. The authors would also like to thank J. Armitstead, J. Cossey and The Bullen Primate Research Foundation for their valuable help with this study.

\section{References}

1. Orchard T, Dorma J, Maser R et al. (1990) Factors associated with avoidance of severe complications after 25 years of IDDM. Pittsburgh Epidemiology of Diabetes Complications Study I. Diabetes Care 13: 741-747

2. Clarke C Neuropathy. In: Werther GA and Court JM (eds), Diabetes and the Adolescent. Miranova Publishers, Camberwell, pp 175-189 
3. DCCT (1988) Factors in development of diabetic neuropathy: Baseline analysis in neuropathy in feasibility phase of diabetes control and complications trial (DCCT). Diabetes 37: 476-481

4. Cameron NE, Cotter MA (1997) Metabolic and vascular factors in the pathogenesis of diabetic neuropathy. Diabetes 46 [Suppl 2]:S31-S37

5. Ward J (1997) Upright posture and the microvasculature in human diabetic neuropathy: a hypothesis. Diabetes 46 [Suppl 2]: S94-S97

6. Ward J (1999) Improving Prognosis in Type 2 Diabetes: Diabetic neuropathy in trouble. Diabetes Care 22: B84-B88

7. Low PA, Nickander KK, Tritschler HJ (1997) The roles of oxidative stress and antioxidant treatment in experimental diabetic neuropathy. Diabetes 46 [Suppl 2]: S 38-S 42

8. Amthor KF, Dahl-Jorgensen K, Berg TJ et al. (1994) The effect of 8 years of strict glycaemic control on peripheral nerve function in IDDM patients: the Oslo Study. Diabetologia 37: $579-584$

9. Ringel RE, Chalew SA, Armour KA et al. (1993) Cardiovascular reflex abormalities in children and adolescents with diabetes mellitus. Diabetes Care 16: 734-741

10. Sugimura K, Dyck PJ (1981) Sural nerve myelin thickness and axis cylinder caliber in human diabetes. Neurology 31: 1087-1091

11. Britland ST, Young RJ, Sharma AK, Clarke BF (1990) Association of painful and painless diabetic polyneuropathy with different patterns of nerve fibre degeneration and regeneration. Diabetes 39: 898-908

12. Malik RA (1997) The pathology of human diabetic neuropathy. Diabetes 46 [Suppl 2]:S50-S53

13. Malik RA (1998) The Primary Pathology and Pathogenesis of Human Diabetic Neuropathy. Diabetologia 41 [Suppl 1] A50 (Abstract)

14. Malone JI, Lowitt S, Korthals JK, Salem A, Miranda C (1996) The effect of hyperglycemia on nerve conduction and structure is age dependent. Diabetes 45: 209-215

15. Brownlee M, Cerami A, Vlassara H (1988) Advanced glycosylation end products in tissue and the biochemical basis of diabetic complications. N Engl J Med 318: 1315-1321

16. Vlassara H (1997) Recent progress in advanced glycation end products and diabetic complications. Diabetes 46 [Suppl 2]:S19-S25

17. Brownlee M, Vlassara H, Kooney A, Ulrich P, Cerami A (1986) Aminoguanidine prevents diabetes-induced arterial wall protein cross-linking. Science 232: 1629-1632

18. Yagihashi S, Kamijo M, Baba M, Yagihashi N, Nagai K (1992) Effect of aminoguanidine on functional and structural abnormalities in peripheral nerve of STZ-induced diabetic rats. Diabetes 41 : 47-52

19. Miyauchi Y, Shikama H, Takasu T et al. (1996) Slowing of peripheral motor nerve conduction was ameliorated by aminoguanidine in streptozocin-induced diabetic rats. Eur J Endocrinol 134: 467-473

20. Cameron NE, Cotter MA (1996) Rapid reversal by aminoguanidine of the neurovascular effects of diabetes in rats: modulation by nitric oxide synthase inhibition. Metabolism 45: $1147-1152$

21. Schmidt RE, Dorsey DA, Beaudet LN et al. (1996) Effect of aminoguanidine on the frequency of neuroaxonal dystrophy in the superior mesenteric sympathetic autonomic ganglia of rats with streptozocin-induced diabetes. Diabetes 45: 284-290

22. Dewhurst M, Omawari N, Tomlinson DR (1997) Aminoguanidine: effects on endoneurial vasoactive nitric oxide and on motor nerve conduction velocity in control and streptozotocin-diabetic rats. Br J Pharmacol 120: 593-598

23. Heffernan S, Phippard A, Sinclair A et al. (1995) A baboon (Papio hamadryas) model of insulin-dependent diabetes. J Med Primatol 24: 29-34
24. Maclean JM, Phippard AF, Garner MG et al. (1987) Group housing of hamadryas baboons: a new cage design based upon field studies of social organization. Lab Anim Sci 37: 89-93

25. Holmes KA, Paull MD, Birrell AM et al. (1996) A unique design for ease of access and movement of captive Papio hamadryas. Lab Anim 30: 327-331

26. Beaven MA, Gordon JW, Jacobsen S, Severs WB (1969) A specific and sensitive assay for aminoguanidine: its application to a study of the disposition of aminoguanidine in animal tissue. $\mathrm{J}$ Pharmacol Exp Ther 165: 14-22

27. Kimura J (1980) Principles of nerve conduction studies. Electrodiagnosis in Diseases of nerves and muscle; Principles and practice. FA Davis, Philadelphia, pp 83-103

28. Phippard A, Maclean J, Garner M, Horvath J (1987) Systemic und pulmonary haemodynamics during continuous ketamine infusions in baboons. Australian Primatology 2: 4-6

29. Goosen DJ, Davies JH, Maree M, Dormehl IC (1984) The influence of physical and chemical restraint on the physiology of the chacma baboon (Papio ursinus). Journal of Medical Primatology 13: 339-351

30. Ansselin AD, Davey DF (1993) The regeneration of axons through normal and reversed nerve grafts. Restorative neurology and neuroscience 5: 225-240

31. Gundersen HJG (1977) Notes on the estimation of the numerical density of arbitary profiles: The edge effect. J Microsc 111: 219-223

32. Howard CF Jr (1982) Nonhuman primates as models for the study of human diabetes mellitus. Diabetes 31: 37-42

33. Yasuda H, Harano Y, Kosugi K et al. (1984) Development of early lesions of microangiopathy in chronically diabetic monkeys. Diabetes 33: 415-420

34. Cornblath DR, Hillman MA, Striffler JS, Herman CN, Hansen BC (1989) Peripheral neuropathy in diabetic monkeys. Diabetes 38: $1365-1370$

35. Jakobsen J, Sidenius P (1985) Nerve morphology in experimental diabetes. Clin Physiol [Suppl 5]: S 9-S 13

36. Sharma AK, Bajada S, Thomas PK (1981) Influence of streptozotocin-induced diabetes on myelinated nerve fibre maturation and on body growth in the rat. Acta Neuropathol (Berl) 53: 257-265

37. Llewelyn JG, Gilbey SG, Thomas PK et al. (1991) Sural nerve morphometry in diabetic autonomic and painful sensory neuropathy. A clinicopathological study. Brain 114: 867-892

38. Donaghue KC (1998) Autonomic neuropathy: diagnosis and impact on health in adolescents with diabetes. Horm Res 50 [Suppl 1]: S33-S37

39. Alteon (1998) Database: www.alteonpharma.com

40. Way KJ, Reid JJ (1994) Effect of aminoguanidine on the impaired nitric oxide-mediated neurotransmission in anococcygeus muscle from diabetic rats. Neuropharmacology 33: $1315-1322$

41. Cameron NE, Cotter MA, Dines K, Love A (1992) Effects of aminoguanidine on peripheral nerve function and polyol pathway metabolites in streptozotocin-diabetic rats. Diabetologia 35: 946-950

42. Wada R (1999) Only limited effects of aminoguanidine treatment on peripheral nerve function, $(\mathrm{Na}, \mathrm{K})$-ATpase activity and thromobomodulin expression in streptozotocin-induced diabetic rats. Diabetologia 42: 743-747

43. Hammes HP, Strodter D, Weiss A et al. (1995) Secondary intervention with aminoguanidine retards the progression of diabetic retinopathy in the rat model. Diabetologia 38: 656-660

44. Foote EF, Look ZM, Giles P, Keane WF, Halstenson CE (1995) The pharmacokinetics of aminoguanidine in end-stage renal disease patients on hemodialysis. Am J Kidney Dis 25: 420-425 